\title{
LE THÉÂTRE DANS LA FORMATION ORATOIRE DES ÉCOLIERS AU XVI SIÈCLE
}

\begin{abstract}
Katell LAVÉANT*
RÉSUMÉ : On a longtemps ignoré la place du théâtre comme outil pédagogique et didactique dans les petites et grandes écoles aux $\mathrm{XV}^{\mathrm{e}}$ et $\mathrm{XVI}^{\mathrm{e}}$ siècles, et le rôle des pratiques dramatiques dans la formation des jeunes gens dans un contexte plus large de sociabilité urbaine. Or on peut analyser ces deux phénomènes en croisant de nombreux documents d'archives (municipales notamment) et littéraires (en particulier des textes de pièces). Leur étude démontre l'ampleur du recours au théâtre comme moyen de formation des jeunes gens qui se destinent aux métiers de la parole à la fin du Moyen Âge.

MoTS-CLÉs : théâtre, enseignement, Moyen Âge, pédagogie, écoles.

\section{DRAMA IN THE TEACHING OF RHETORICAL ARTS IN FRENCH SCHOOLS IN THE $15^{\mathrm{TH}}$ AND $16^{\mathrm{TH}}$ CENTURIES}

ABSTRACT: The importance of drama as pedagogic and didactic tool in the $15^{\text {th }}$ and $16^{\text {th }}$ century schools has long been ignored, and so has the role of theatrical practices in the education of young men in the context of urban sociability. Yet those two aspects can be analyzed thanks to numerous historical sources (municipal archives) and literary ones (plays contextualized thanks to archive data). Their study shows how often drama was used as a tool in the first stages of the training of men aspiring to start a career in rhetorical arts.
\end{abstract}

KEYWORDS : drama, education, Middle Ages, pedagogy, schools.

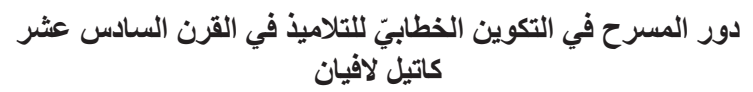

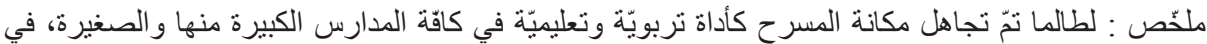

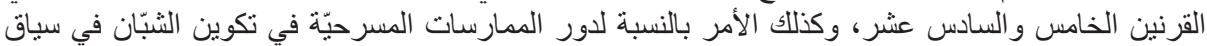

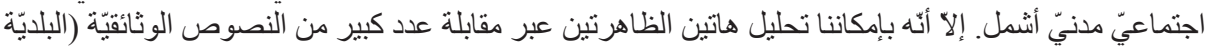

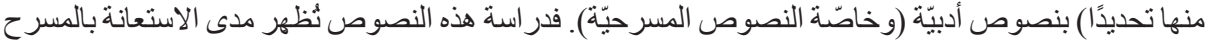

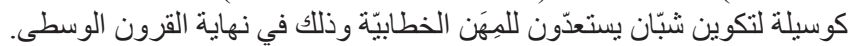

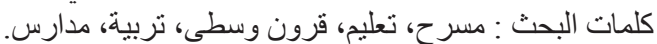

* Katell Lavéant, née en 1978, est enseignante-chercheuse aux universités d'Utrecht et d'Amsterdam. Ses recherches portent sur le théâtre à la fin du Moyen Âge et au XVI ${ }^{\text {e }}$ siècle (textes et culture dramatique). Elle a notamment publié Un théâtre des frontières. La culture dramatique dans les provinces du Nord aux XV et XVI siècles (Orléans, Paradigme, 2011). Adresse : Universiteit Utrecht, FGW - Departement Moderne Talen, Trans 10, 3512 JK Utrecht, Pays-Bas (k.laveant@uu.nl). 


\section{DIE ROLLE DES THEATERS IN DER ORATORISCHEN AUSBILDUNG VON SCHÜLERN IM 16. JAHRHUNDERT}

ZuSAMMENFASSUNG : Lange Zeit blieben die Bedeutung des Theaters als pädagogisches und didaktisches Instrument der ,petites écoles “ und,,grandes écoles “im 15. und 16. Jahrhundert sowie die Rolle des Schauspiels in der Ausbildung junger Menschen im Kontext städtischer Geselligkeit unbeachtet. Dabei lassen sich diese beiden Phänomene durchaus untersuchen, wenn man die Analyse von Archivtexten (insbesondere aus Stadtarchiven) und literarischen Werken (besonders Theatertexte) miteinander kombiniert. Die Analyse verdeutlicht das Ausmaß, zu dem das Theater gegen Ende des Mittelalters eingesetzt wurde, um jungen Menschen das Reden in der Öffentlichkeit als zentrale Fertigkeit für ihren zukünftigen Beruf zu vermitteln.

SCHLAGWÖRTER : Theater, Unterricht, Mittelalter, Pädagogik, Schulen.

\section{EL TEATRO EN LA FORMACIÓN ORATORIA DE LOS ESCOLARES DEL SIGLO XVI}

RESUMEN : Hemos ignorado durante largo tiempo el lugar del teatro como herramienta pedagógica y didáctica en las pequeñas y grandes escuelas de los siglos XV y SVI, y el rol de las prácticas dramáticas en la formación de la gente joven en el contexto más amplio de la sociabilidad urbana. Podemos analizar estos dos fenómenos cruzando los numerosos documentos de archivos (sobretodo municipales) y literarios (en particular los textos de obras teatrales). Su estudio muestra la extensión del recurso teatral como medio de formación de la gente joven que se dirige a profesiones discursivas a finales de la Edad Media.

PALABRAS CLAVE : teatro, enseñanza, Edad Media, pedagogía, escuelas.

戲劇在16世紀學生演講教學中的作

用

卡戴爾・拉維昂

摘要：長期以來，人們都忽視在 15 到16世紀大小學校中戲劇作為教學 和訓導工具的位置, 以及戲劇實踐 在城市社交大背景中對年輕人的教 育作用。我們將結合大量檔案資料 (尤其是市政府的) 和文學作品（ 特別是劇本) 來分析這兩種現象, 并由此揭示出戲劇在教育中世紀末 有志從事演說職業的年輕人時發揮 的重要作用。

關鍵詞: 戲劇, 教育, 中世紀, 教 學法, 學校。

$$
\begin{gathered}
16 \text { 世紀における生徒の弁論教育の } \\
\text { ための演劇 } \\
\text { カテル・ラヴェァン }
\end{gathered}
$$

要約：15世紀から16世紀の大小の学 校において、教育手段としての演劇 の役割は長い間、問題視されなかっ た。また都市における社会性という 広範囲な背景のなかで若者の養育に おいての演劇の役割も同樣である。 われわれは、このょうな現象を、多 くの古文書 (特に市町村のもの) そ して、文学資料（2つの戯曲資料） を交差させながら分析していきた い。本文はとりわけ、中世後期、発 言と関わる仕事を目指す若者の育成 方法のひとつとしての演劇の駆使の 重要性を証明する。

キーワード：演劇、教育、中世、 教育学、学校。 
E ntre 1556 et 1560, deux séries de gravures de Pieter Bruegel l'Ancien sur les sept péchés capitaux et les sept vertus catholiques furent imprimées à Anvers par Hieronymus Cock. Dans l'une de ces gravures (Temperantia), ce sont les sept arts libéraux qui entourent l'allégorie de la tempérance, indiquant ainsi que celui qui a été formé à ces sept arts est particulièrement à même de faire preuve de cette vertu. On y voit entre autres des enfants de chœur dirigés par des maîtres de chant pour illustrer l'apprentissage de la musique, tandis qu'un groupe d'enfants assis autour d'un maitre et lisant un livre s'exerce à la maîtrise de la grammaire. La rhétorique est quant à elle symbolisée dans le coin supérieur gauche par un groupe d'acteurs sur des tréteaux, jouant une pièce devant des spectateurs que l'on voit de dos.

La manière de représenter ici l'activité théâtrale n'est pas nouvelle : elle s'inscrit dans une tradition iconographique bien établie au Moyen Âge, dans laquelle la vision de l'espace scénique est codifiée à l'aide d'un certain nombre d'éléments récurrents (notamment des acteurs séparés du public par une scène en hauteur, au niveau de la tête des spectateurs et la présence d'un rideau de fond créant des coulisses) ${ }^{1}$. Dans la gravure de Bruegel, deux acteurs jouent sans doute le rôle de personnages allégoriques, étant donné la présence d'un phylactère sur le vêtement de l'un des deux, qui permet traditionnellement au public d'identifier la nature de l'allégorie représentée, mais un troisième personnage est visiblement un sot, comme l'indiquent son bonnet et sa marotte. Il s'agit donc probablement d'une pièce à valeur morale et didactique, mais de laquelle l'élément joyeux n'est pas absent.

Il est extrêmement significatif que, pour illustrer l'apprentissage de l'art libéral lié à la maîtrise de la parole, Bruegel choisisse de dépeindre des acteurs de théâtre en action ${ }^{2}$. De fait, dans un espace flamand dans lequel les membres des chambres de rhétorique (les rederijkers) prennent de plus en plus d'importance dans le cadre urbain au XVI ${ }^{\mathrm{e}}$ siècle, grâce notamment à leurs activités dramatiques, c'est apparemment le théâtre qui s'impose naturellement à l'artiste comme la meilleure représentation iconographique de l'art rhétorique. La combinaison dans la même gravure de cette scène théâtrale avec des situations d'apprentissage dans un cadre ecclésiastique pour les jeunes enfants (enfants de chœur notamment) nous oriente alors vers une piste encore peu explorée : le théâtre y apparaît comme un moyen pédagogique et didactique de maîtriser la compétence rhétorique attendue des élèves des petites et grandes écoles héritées du système médiéval. Cette inscription dans le cursus scolaire est bien plus précoce qu'on ne le pensait jusqu'à présent, et précède de beaucoup son utilisation à des fins pédagogiques dans les collèges humanistes et chez les Jésuites ${ }^{3}$. En ce sens, le théâtre comme moyen pédagogique n'est pas une innovation du milieu du

1. Sur cette tradition iconographique, voir Rousse, 2004.

2. Cette association sera d'ailleurs reprise par d'autres artistes flamands. Ainsi, dans sa série sur les sept arts libéraux, Cornelis Cort (1533?-1578), montre en arrière-plan d'une femme symbolisant la Rhétorique une place sur laquelle une pièce est en train d'être jouée sur des tréteaux similaires à ceux dépeints par Bruegel, et par bien d'autres. Sur l'iconographie flamande, voir l'article (accompagné d'illustrations) d'HuMmelen, 1989.

3. Sur le théâtre des collèges, voir BouhaÏK-Gironès, 2011b ; Ferrand, 2009 et 2010. Sur le théâtre des Jésuites, voir Dainville, 1978 ; Valentin, 2001 ; Olaizola Sanchez, 2005. 
$\mathrm{XVI}^{\mathrm{e}}$ siècle, mais bien de la reprise de traditions pédagogiques qui existaient déjà à la fin du Moyen Âge.

Nous nous proposons d'éclairer ici cet aspect encore très peu mis en avant dans les études sur la formation scolaire à cette époque ${ }^{4}$, et de souligner la place du théâtre dans la formation des enfants et jeunes gens. Ceci implique de réfléchir à la fois au cadre et au contenu précis de l'enseignement primaire aux $\mathrm{XV}^{\mathrm{e}}$ et $\mathrm{XVI}^{\mathrm{e}}$ siècles. Une enquête complète devrait nous amener à examiner ce rôle du théâtre dans la formation d'écoliers appelés à exercer différents métiers de la parole, du prédicateur à l'avocat, du maître d'école ou d'université au maire ou à l'échevin ${ }^{5}$. Dans l'espace qui nous est ici imparti, nous devrons restreindre notre propos à un exemple, celui de la formation des élites urbaines exerçant des fonctions dans le cadre municipal, en nous appuyant sur l'exemple des villes francophones du sud des Pays-Bas. Il existe en effet pour ces villes un certain nombre de sources et d'études qui permettent d'ouvrir de nouvelles pistes de réflexion pour l'espace francophone. Bien que nous nous penchions ici uniquement sur la première phase du cursus scolaire, avant le moment où les étudiants orientent leurs études vers une carrière spécifique, un certain nombre de nos observations pourront servir de point de départ à une enquête similaire pour d'autres métiers de la parole. Nous montrerons également l'intérêt de ne pas limiter cette enquête uniquement à l'enseignement dans le cadre scolaire proprement dit, mais également d'envisager la formation des jeunes gens au sens plus large. On verra ainsi en quoi la pratique dramatique peut jouer un rôle dans les premiers stades de formation et d'apprentissage des jeunes gens dans un contexte plus large de sociabilité urbaine.

\section{LE THÉÂTRE COMME OUTIL D'ENSEIGNEMENT DANS LES ÉCOLES AU XVI SIÈCLE}

\section{Qu'entend-on par « école » à la fin du Moyen Âge?}

L'organisation de l'enseignement primaire et secondaire à la fin du Moyen Âge a moins attiré l'attention des chercheurs que celle de l'enseignement universitaire ${ }^{6}$, sans doute du fait de sources beaucoup plus clairsemées et parcellaires. On s'accorde

4. Par le terme d'« école », nous désignons ici essentiellement les petites et grandes écoles capitulaires ou privées en milieu urbain, puisque nous centrons notre étude sur ces centres d'enseignement primaire hérités du Moyen Âge. Nous ne proposons donc pas d'analyse exhaustive du théâtre dans les collèges humanistes les plus célèbres ni dans ceux des Jésuites, bien que nous y référions parfois à titre de comparaison (exemples de Cambrai et Lyon notamment). Ceux que nous nommons ici « écoliers » sont les élèves de ces écoles (souvent appelés « enffans » dans les sources) et non les étudiants plus âgés fréquentant l'université, bien que le terme escolier désigne encore ces derniers à la fin du Moyen Âge.

5. Pour la formation des maîtres d'école, on peut suggérer que ces derniers utilisent avec leurs élèves les techniques qu'ils ont eux-mêmes acquises grâce au théâtre, et qui témoignent en tout cas qu'ils connaissaient bien les pièces du répertoire médiéval : voir LAVÉANT, 2011b. Sur la formation des avocats, signalons le rôle de la Basoche : voir BouHAïK-GIRonÈs, 2007. Pour une réflexion sur la maîtrise de la rhétorique et de l'art oratoire des avocats grâce au théâtre, voir BouHAÏK-GIRONĖs, 2011a.

6. Pour lequel on rappellera les travaux de Jacques Verger, synthétisés notamment dans VERGER, 1995 et 1999. 
cependant aujourd'hui à reconnaître la mise en place au cours du Moyen Âge de deux types d'écoles : la petite, pour des enfants âgés de 6 à 10 ou 12 ans et s'initiant aux savoirs élémentaires (maîtrise de la lecture, de l'écriture, du calcul) ; et la grande, pour des enfants de 10 ou 12 à 16 ans, qui y apprennent le latin et les bases des sept arts libéraux. Le cadre est d'abord ecclésiastique, les écoles relevant de l'autorité des chapitres locaux, d'où l'assimilation fréquente des élèves aux enfants de chœur, et le statut double de leur enseignant, à la fois maître d'école et maître de chapelle. Ce n'est que dans un deuxième temps que des écoles laïques, tenues par des clercs mais financées par les municipalités, font leur apparition, à partir du XIII ${ }^{\mathrm{e}}$ siècle. À ce titre, on constate dans l'ensemble des Pays-Bas une sécularisation de l'enseignement assez précoce, due à l'influence des nombreux marchands souhaitant faire donner à leurs enfants une éducation plus adaptée à leurs besoins ${ }^{7}$. Pour les villes que nous allons étudier, Alain Derville a ainsi montré qu'il existait à la fin du Moyen Âge beaucoup plus d'écoles (privées) qu'on ne l'a dit jusqu'à présent ${ }^{8}$. Les organisations duales petite/grande école et école ecclésiastique/laïque que l'on observe pour les Pays-Bas ne diffèrent pas fondamentalement de ce qui a été décrit dans plusieurs villes françaises et dans d'autres régions francophones, comme le montrent des études sur Reims, Soissons ou encore la Suisse Romande ${ }^{9}$.

Si l'organisation globale du système d'enseignement se dessine donc avec une relative netteté, il reste un certain nombre de problèmes concernant les connaissances précises que l'on peut acquérir sur ces écoles. Faut-il ainsi distinguer au $\mathrm{XVI}^{\mathrm{e}}$ siècle, sur le plan de l'organisation comme des méthodes pédagogiques, les grandes écoles des collèges qui apparaissent en nombre à cette époque ? On verra avec des exemples de mentions d'archives que les sources sont souvent ambiguës sur le statut des lieux d'enseignement cités, qui ne sont pas toujours inscrits dans l'une ou l'autre des catégories, au moins avant que l'apparition des collèges de Jésuites ne soit plus clairement renseignée par les documents d'archives. Autre question devenue cruciale à la fin du Moyen Âge, à mesure que le nombre des élèves augmente : quelle est la finalité de ces études élémentaires pour la majorité des élèves qui ne poursuivra pas des études universitaires et qui n'est pas destinée, par les familles, à une carrière ecclésiastique ? Cette donnée influe-t-elle sur le contenu de l'enseignement dispensé, quand ce dernier est financé par les municipalités et non par les chapitres ? Peut-on alors aller plus loin dans l'appréhension des textes utilisés pour l'enseignement, en particulier dans les petites écoles pour lesquelles les renseignements sont plus limités que pour les grandes, hors des textes identifiés jusqu'à présent (pour les petites écoles, essentiellement les principales prières et les répons de la messe pour le catéchisme) ? Il nous semble que par le biais de l'étude du théâtre, on peut proposer des hypothèses qui permettent d'aller plus loin pour répondre à ces questions que ne l'autorisent les sources traditionnellement invoquées dans le dossier des écoles.

7. De Ridder-Symoens, 1995b : cet article propose une synthèse et une bibliographie très fournies sur la question. Voir également, pour des éléments synthétiques sur l'organisation de l'enseignement aux Pays-Bas : De RidDer-Symoens, 1995a et 2004.

8. Derville, 1984.

9. Desportes, 1975 ; Carolus-Barré, 1975 ; Pibiri, 1998, p. 145-149. 


\section{Quelles sources historiques pour l'histoire du théâtre à l'école?}

Pour documenter l'utilisation du théâtre dans les écoles, nous disposons d'un grand nombre de mentions d'archives qui signalent la représentation, en public ou devant des spectateurs choisis, de pièces de théâtre par des écoliers sous la direction de leur maître. Pour les villes du sud des Pays-Bas, notamment, on dispose de mentions dans les livres de compte des villes de Béthune, Cambrai, Douai, Lille ou encore Saint-Omer, qui montrent que des récompenses sont accordées à des maîtres d'école ${ }^{10}$. Il est difficile, d'après ces seules mentions, de saisir comment le théâtre est alors intégré à l'enseignement au sein de ces écoles, mais on peut au moins comprendre comment fonctionne le système de représentation des pièces, et analyser la valeur pédagogique de ces spectacles. Nous voulons ici étudier le cadre des représentations (dates et lieux), ce que l'on peut savoir du contenu des pièces, la langue dans laquelle les écoliers se produisent, les types de public à envisager pour ces représentations, et les indices renvoyant à une implication des maîtres dans la culture dramatique, sans oublier la question de la participation des femmes (élèves et enseignantes) aux représentations.

Il ressort ainsi de ces documents d'archives que les écoliers mentionnés dans les comptes sont le plus souvent élèves d'écoles capitulaires et que leur maître les prépare et les dirige pour des représentations de pièces en français ou en latin, à des dates variées mais souvent significatives du calendrier : la période de Carnaval à Béthune, le 10 février, jour de sainte Scolastique, à Cambrai, la mi-carême ou l'Ascension, par exemple. On sait en effet que les pièces de théâtre proposées au public des villes à cette époque, que ce soient les mystères ou les pièces des groupes appelés compagnies joyeuses, le sont souvent selon un calendrier qui combine les grandes dates du calendrier liturgique et celles du calendrier festif de l'hiver (période de Carnaval mais aussi de Noël) ${ }^{11}$. Les écoliers ne dérogent pas à ce système, et l'on peut constater à travers les dates indiquées dans les sources que l'accent est là aussi mis sur l'un des deux aspects, festif ou religieux (sans d'ailleurs, comme on va le voir, que la tonalité du jour choisi détermine celle des pièces jouées à cette occasion). De plus, il est significatif que, quand on possède des mentions régulières dans une ville, ces dernières laissent à penser que l'on a affaire à une fête annuelle (c'est le cas à Cambrai, par exemple). Or les villes de la région connaissent d'autres fêtes annuelles, qui sont le fait des membres de la partie basse de la hiérarchie ecclésiastique (enfants de chœur, mais aussi vicaires plus âgés), à l'occasion des fêtes de l'Avent et du début de l'année (fête des Innocents, des Fous ou des Ânes), et aussi des compagnies joyeuses, nombreuses dans la région, qui proposent chaque année des festivités auxquelles elles se convient d'une ville à l'autre, et qui sont subventionnées par la municipalité ${ }^{12}$. Il est donc intéressant de voir que les représentations des écoliers complètent ce calendrier dramatique qui rythme le cours de l'année, quand du moins on peut reconstruire la fréquence de ces représentations d'après les mentions d'archives. Ainsi, pour Cambrai, par exemple, la principale compagnie joyeuse de la ville, l'abbaye de Lescache-Pourfit (« ceux qui

10. Les exemples cités dans ce paragraphe sont tirés des articles suivants : LA Fons DE MÉLICOCQ, 1848 et 1886-1887 ; DURIEUX, 1883 ; BORGNET, 1868-1869.

11. LAVÉANT, 2011c, p. 139-156.

12. LAVÉANT, 2011c, p. 157-172. Sur les Fous et les Innocents, voir notamment DAHHAOUI, 2005. 
cachent leur profit », un nom ironique destiné à souligner une prétendue pingrerie ou au contraire un prétendu manque d'argent de ses membres), organise sa fête le jour du Vingtième (vingtième jour après la naissance du Christ), le 13 janvier. Les représentations scolaires le jour de sainte Scolastique, le 10 février, peuvent alors être vues comme une prise de relais des représentations dans la ville par un autre type d'acteurs. Notons d'ailleurs qu'au moins à Douai, dans la première moitié du XVI ${ }^{\mathrm{e}}$ siècle, les activités festives des écoliers semblent se confondre avec celles des compagnies joyeuses, puisqu'on trouve une compagnie des Bons Enfants et leur évêque recteur (active de

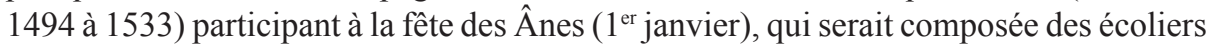
de la maison des Bons Enfants de l'église Saint-Amé ou saint-Pierre ${ }^{13}$.

Les élèves se produisent sur la place publique (c'est le cas à Béthune) ou seulement devant les échevins (« en chambre », c'est-à-dire dans un lieu clos, par exemple une pièce de l'hôtel de ville, comme c'est le cas à Cambrai et à Lille). C'est parce que cette représentation a un caractère public ou est organisée pour le profit de représentants de la municipalité que le maître reçoit une récompense payée sur les deniers publics, petite somme d'argent ou don en vin, ce qui explique que l'on en ait conservé une trace dans les archives de la ville. Les pièces représentées ont des sujets variés. Comme on peut s'y attendre, certaines mentions indiquent des pièces morales, par exemple sur le thème de l'Annonciation de la Vierge Marie (Béthune, 1546), la moralité et le martyre de saint Lambert ou un épisode biblique sur la vie du roi Salomon (Dinant, 1563). Cependant, le plus souvent, le sujet de la pièce n'est pas mentionné et il faut se contenter de descriptions plus vagues, telles « un moral » ou « une histoire » (terme renvoyant à une pièce à sujet historique ou biblique). Pour autant, le répertoire est varié et ne se limite pas au théâtre religieux et moral, puisqu'on trouve aussi souvent la mention de farces et, dans la deuxième moitié du $\mathrm{XVI}^{\mathrm{e}}$ siècle, de comédies. On retrouve également dans certaines descriptions l'association dans une même pièce ou série de pièces des deux registres, moral et joyeux, telle qu'elle était dépeinte dans la gravure de Bruegel, comme le montre le cas d' « un ju moral avecq la farse joieuse » à Béthune en 1546. Comme nous l'avons souligné, la date à laquelle est jouée la pièce n'a pas nécessairement d'influence sur sa tonalité : les écoliers de Béthune peuvent ainsi jouer le dimanche de la Passion 1571 « en langue lattine une histoire avecq certaine farce », tandis que ceux de Cambrai représentent régulièrement des comédies le jour de sainte Scolastique.

Les archives indiquent également que la langue dans laquelle est donnée la représentation varie : souvent, elle n'est pas indiquée, mais un certain nombre de mentions précisent quand la pièce est jouée en latin. Faut-il alors en conclure que si la précision ne s'impose pas, la pièce a été jouée en français ? On a en tout cas au moins un cas de représentation mixte : à Dinant, en 1563, le martyre de saint Lambert est joué en latin, mais l'histoire de Salomon est donnée en français (« en wahlon »). Concernant la langue, il est de tradition de distinguer un enseignement en français dans les petites écoles et en latin dans les grandes écoles (et les universités ${ }^{14}$ ). Faudrait-il donc partir

13. Guilleray, 2002, p. 115.

14. Il faudrait cependant s'interroger de nouveau sur la validité d'une distinction aussi tranchée, comme l'indique BouHAÏK-GIRONÈs, 2011a, p. 235-236. 
de cette distinction pour établir la catégorie d'âge des élèves et considérer que ce sont les élèves de la grande école qui jouent les pièces en latin, quand les mentions que l'on a conservées le précisent ? Faudrait-il également partir de ce constat pour, à l'inverse, déterminer dans quelle langue jouent les élèves, quand il est fait mention de leur classe d'âge, comme c'est le cas à Béthune en 1525, quand Pierre de Manchicourt, « maistre de la grande escolle », fait jouer à ses élèves « certains jus [jeux] et esbatemens par lui et ses enffans escolliers faits au-devant de la halle le jour du dimanche gras »? Les élèves ont-ils pu dans ce cas jouer une pièce en latin devant un large public ? Cela n'est pas inconcevable, puisqu'en 1579, dans la même ville, des élèves jouent bien « durant la procession générale, sur ung eschaffault une ystoire, en langue latine ", mais il serait hâtif d'en conclure que les petits enfants jouent systématiquement en français, et les élèves plus âgés en latin. De fait, l'exemple de Barthélémy Aneau, qui fait jouer une pièce en français aux élèves plus âgés de son collège en 1541 plutôt que de suivre les nouvelles prescriptions des humanistes sur la nécessité de privilégier le latin comme langue de l'enseignement ${ }^{15}$, montre qu'il faut plutôt traiter ces situations au cas par cas, quand les informations que l'on possède sont suffisamment détaillées pour pouvoir proposer des conclusions fermes à ce sujet. Il apparaît en tout cas d'après l'exemple de la représentation en 1579 à Béthune qu'une représentation en latin pour un large public ne semble pas poser de problème. Il est possible d'envisager que ce public comprend mieux le latin que l'on ne pourrait le penser de prime abord, comme en témoignent aussi les nombreux sermons joyeux et farces qui proposent des jeux de mots sur le latin (de messe ou macaronique) ${ }^{16}$.

De fait, on peut envisager deux types de public. Quand la représentation a lieu sur la place principale de la ville, il est certain qu'elle peut attirer un large public, en particulier quand elle a lieu à l'occasion d'autres festivités telles que les fêtes des compagnies joyeuses et les processions. Mais souvent, il est précisé que la représentation a lieu dans un cadre plus restreint, devant les échevins. Ces deux données nous orientent vers les deux interprétations possibles du but de ces spectacles. Comme le rappelle le compte de 1546 à Béthune, le maître d'école fait jouer ses élèves « pour récréer le peuple et enseignier les josnes enffans ». Il s'agit donc d'abord de proposer un spectacle qui, comme ceux des autres groupes de théâtre cités plus haut, offre un divertissement aux habitants de la ville. Mais on peut se demander comment interpréter la deuxième précision : s'agit-il de proposer aux enfants de la ville un spectacle qui leur délivre un enseignement moral, comme pouvait l'être celui offert par les pièces jouées pendant la procession de Lille, dont Alan Knight a montré que les sujets choisis contenaient un message à direction des jeunes gens pour les inciter à régler leurs mœurs ${ }^{17}$ ? Ou faut-il comprendre que les « jeunes enfants » sont les élèves qui se produisent et qui font ainsi l'expérience, en présentant leur pièce à un public nombreux (et averti), de la prise de parole en public ? Dans le deuxième cas, il est probable qu'une représentation devant les échevins comporte également une dimension de divertissement,

15. Doudet, 2011 ; FerRand, 2011.

16. Voir à titre d'exemple Koopmans et Verhuyck, 1987, ainsi que la réflexion de Marie BouhaïkGironès sur l'emploi du latin et du français dans les milieux universitaires et juridiques, d'après l'étude de la Farce de Maître Mimin (BouHAïK-Gironès, 2011a).

17. KNIGHT, 1996. 
certainement doublée pour ces autorités de l'occasion de vérifier le niveau de l'enseignement dispensé et les progrès des élèves, dont on peut penser qu'un certain nombre sont leurs propres enfants.

On doit d'ailleurs certainement envisager le fait que le théâtre n'est pas un simple exercice de « spectacle de fin d'année », de niveau médiocre. Le passage des élèves devant un public habitué à des représentations de qualité sur la place principale de la ville ou aux côtés de groupes expérimentés comme les compagnies joyeuses tend en effet à prouver une certaine exigence sur ce point, comme aussi les indices relevés dans les archives qui indiquent que certains maîtres s'impliquaient dans la vie théâtrale de leur cité, hors du cadre proprement scolaire. On sait ainsi qu'au milieu du Xvi siècle, un maître d'école à Tournai est également membre de la chambre de rhétorique de la ville, dont les écrits poétiques et théâtraux, soupçonnés de sympathies pour la Réforme, sont surveillés de près par les autorités catholiques ${ }^{18}$. De même, le prêtre Antoine Petit est à la même époque maître d'une petite école dans la paroisse Saint-Denis à SaintOmer mais aussi acteur dans plusieurs représentations sur la grand'place ${ }^{19}$.

Enfin, pour compléter ce tour d'horizon des informations que nous livrent les sources d'archives sur les représentations scolaires, il faut citer le cas d'une mention trouvée à Douai : en 1582, du vin est offert à une demoiselle Marie Desmollins, femme de Charles Gigan, clerc assermenté de la ville, et à ses écolières, pour avoir représenté des histoires lors d'une procession ${ }^{20}$. Cette mention confirme les données avancées par Alain Derville pour une scolarisation et un taux d'alphabétisation des filles supérieurs à ce que l'on a coutume d'envisager pour cette époque ${ }^{21}$, et montre également que la participation des femmes au théâtre n'était pas aussi rare qu'on a longtemps bien voulu le croire ${ }^{22}$.

Les analyses proposées ici se fondent sur les archives des Pays-Bas méridionaux, mais nous pensons que des enquêtes plus approfondies dans d'autres domaines français ou francophones permettraient de faire ressortir des résultats similaires, qui montrent le lien fort entre enseignement et représentations théâtrales. On peut ainsi citer des recherches menées en Suisse romande, qui montrent que dans un système d'enseignement dans lequel les maîtres d'école sont engagés par les autorités municipales, les écoliers sont associés aux événements publics. C'est le cas à Yverdon, commune située dans le canton de Vaud, dont les comptes communaux font état des activités théâtrales des écoliers dès le milieu du $\mathrm{Xv}^{\mathrm{e}}$ siècle, autant pour des fêtes de jeunesse (élection du roi des écoliers) que pour des représentations de type officiel, pour distraire des hôtes

18. LAVÉANT, 2011c, p. 258-261.

19. Hirschauer, 1907-1911, p. 507 ; LavÉANt, 2011c, p. 473-475.

20. Information issue des notes de travail de l'érudit Émile Picot, rassemblées dans un Répertoire des villes accompagnant son Répertoire historique et bibliographique de l'ancien théâtre français (souvent désigné par les spécialistes du théâtre médiéval sous le nom de fichier Picot) : Рісот, s.d., p. 290. Pour un inventaire succinct de ce fichier, précieux pour l'histoire du théâtre médiéval, voir BOUHAÏK-GIRONÈs, 2010.

21. DeRVILLe, 1984, p. 765-768.

22. Pour l'ensemble des domaines français et francophones, on trouve des mentions en nombre important indiquant la participation de femmes à des représentations de mystères, leur rôle comme actrices professionnelles dans des troupes itinérantes, ainsi que leur implication dans la subvention de festivités à caractère dramatique : LAVÉANT, 2011c, p. 129 (note), 132, 189-190 et répertoire. 
de passage ou jouer en public des « Miracles de saint Nicolas ${ }^{23}$. Des recherches similaires dans les fonds d'archives d'autres villes et régions permettraient certainement de mettre à jour d'autres cas de théâtre scolaire. On obtient également des informations intéressantes en croisant les sources d'archives avec des sources littéraires, comme on va le voir avec le cas des pièces d'élèves de la petite école du Mans et du collège de la Trinité à Lyon au début du XVI ${ }^{\mathrm{e}}$ siècle.

\section{Quelles sources littéraires pour l'histoire du théâtre scolaire?}

En parallèle aux sources d'archives, il faut en effet prendre en compte quelques exemples de recueils imprimés de pièces de théâtre qui témoignent de l'utilisation de pièces de théâtre à des fins pédagogiques et didactiques dans les écoles. Nous pensons ici à deux cas précis, mais il faudrait mener une enquête complète pour, le cas échéant, répertorier plus de cas similaires. En 1512, François Briand, maître d'école et des enfants de chœur engagé par le chapitre de Saint-Pierre au Mans, fait jouer des pièces lors des dimanches de l'Avent par les « petis enfans » dont il a la charge ${ }^{24}$. On ignore si la représentation a lieu devant un large public en marge des messes de l'Avent ou devant les seuls membres du chapitre, car nous ne disposons pas de documents d'archive sur ce contexte. De fait, c'est grâce à une version de ces pièces imprimée immédiatement après les représentations que nous avons conservé la trace de cette pratique, qui témoigne de l'intérêt, pour leur auteur, de transmettre ses textes à un lectorat plus large que le seul public de ces représentations. Le thème des quatre pièces témoigne des éléments que l'on a vus au travers des mentions d'archives : mélange des registres religieux et joyeux (une pièce sur l'Annonciation et une suivant la liturgie avec une réflexion sur l'Apocalypse reflétant le thème de la messe du deuxième dimanche de l'Avent, en parallèle à une farce et une pièce comique sur les doutes de Joseph quant à la grossesse miraculeuse de Marie) ; inspiration de thèmes et de scènes récurrents du théâtre médiéval que l'auteur connaît visiblement bien (procès de Paradis, Annonciation, doutes de Joseph, opposition comique d'un aveugle et de son valet) ; didactisme des scènes, notamment à travers les nombreuses références aux textes religieux et à la littérature antique employée pour apprendre le latin dans les écoles, proposées dans l'imprimé sous forme de gloses. On constate alors la relative complexité de ces pièces pour de jeunes acteurs, à la fois compte tenu des thèmes et des nombreuses références à maîtriser pour les acteurs comme pour le public, et du fait des aspects spécifiquement dramaturgiques (la mise en scène de la farce, notamment, nécessite une maîtrise non négligeable des ressorts de l'action comique). Cependant, il faut aussi souligner en parallèle qu'une pièce au moins, la dernière à être représentée, nous paraît être destinée à des acteurs plus jeunes, notamment du fait de sa dramaturgie plus simple et de son

23. PIBIRI, 1998, p. 145-149.

24. Le titre complet de l'imprimé contenant ces pièces ainsi que des partitions et textes pour des Noëls chantés est le suivant : «Se ensuyvent les nouelz nouvaulx de ce present an mil cinq cens et douze dont en y a plusieurs notez a deux parties dont l'une n'est que le plain chant. Avecques quatre histoires par personnaiges sur quatre evangilles de l'Advent a jouer par les petis enfans les quatre dimenches dudit Advent. Composez par maistre Françoys Briand, maistre des escolles de Sainct Benoist en la cité du Mans ». Sur Briand et pour l'ensemble de cette présentation, voir LAVÉANT, 2011a et 2011b. 
contenu plus facilement mémorisable. Joseph y dresse notamment une longue liste de ses outils de menuisier, véritable exercice mnémotechnique, mais peut-être aussi rappel de connaissances directement utiles à des élèves se destinant à ce métier ou à un métier de clerc appelé à fréquenter des artisans et à connaître leur environnement de travail ${ }^{25}$. On voit donc par cet exemple qu'il ne faut pas seulement analyser les textes des pièces dans leur dimension pédagogique sur le plan de l'apprentissage de la parole, mais aussi se poser la question de leur prise avec la réalité professionnelle qui attend les élèves à leur sortie de l'école.

Barthélémy Aneau, recteur au collège de Lyon, s'inscrit dans cette tradition du théâtre de l'Avent quand il fait jouer et chanter à ses élèves des Noëls et un mystère rassemblés dans un volume intitulé Chant natal (1539) ainsi qu'une moralité, le Lyon marchant (1541). Là encore, les textes sont publiés tout de suite après les représentations, qui ont eu lieu dans l'enceinte du collège mais dont on peut penser qu'elles ont été vues par les notables de la ville ${ }^{26}$. Comme pour Briand et les mentions d'archives étudiées, on constate une inscription dans le calendrier religieux et festif en ce qui concerne la thématique du Chant natal, tandis que la moralité du Lyon marchant, en proposant un discours crypté sur les événements du temps, témoigne que les thèmes abordés par le théâtre scolaire s'étendent à l'actualité des débats politiques locaux et nationaux. Enfin, il faut signaler l'emploi du français comme langue de jeu, certes en accord avec les préceptes de l'auteur du Quintil Horatian, là où l'on pourrait s'attendre plutôt à la prépondérance du latin de la part d'un recteur humaniste.

Qu'ajoutent ces documents à la compréhension que nous avons du phénomène du théâtre scolaire ? D'abord, l'accès au texte est essentiel pour mieux en saisir les enjeux pédagogiques et didactiques, et ces pièces complètent heureusement les mentions d'archives, trop lacunaires sur le plan du contenu de l'enseignement. On y saisit notamment l'importance du théâtre comme moyen privilégié de l'apprentissage de la prise de parole en public, de l'entraînement de la mémoire et de la pronuntiatio ou action oratoire ${ }^{27}$. De plus, la publication rapide de ces textes après la représentation et leur utilisation possible par un public différent de celui de l'enseignement (comme en témoigne la conservation d'un exemplaire des pièces de Briand dans un monastère, probablement à des fins de lecture stimulant la méditation religieuse) prouvent que leurs auteurs ne considéraient pas ces pièces comme de simples exercices scolaires devant rester cantonnés à l'intérieur de l'école, mais bien comme des textes offrant une valeur certaine pour tout lecteur s'intéressant à l'écriture dramatique comme au contenu multiple proposé. L'étude de ces textes littéraires est donc essentielle pour saisir l'importance du théâtre dans la pratique scolaire.

25. LAVÉANT, 2011b, p. 260-261.

26. Les données présentées sur le théâtre de Barthélémy Aneau proviennent de DoudEt, 2011 ; FERRAND, 2011 et LAVÉANT, 2011a.

27. FerRAND, 2010 ; BouHAÏK-Gironès, 2011 a. 


\section{AU-DELÀ DU SYSTÈME SCOLAIRE \\ LE THÉÂTRE ET LA FORMATION DES FUTURES ÉLITES URBAINES}

Si nous avons insisté jusqu'ici sur le cadre scolaire, il nous semble cependant qu'il ne faut pas se limiter exclusivement à cet environnement pour saisir le rôle éducatif du théâtre dans les villes de la fin du Moyen Âge, au risque de n'en saisir que l'aspect pédagogique le plus évident. Or c'est en élargissant le propos au rôle formateur du théâtre dans d'autres structures sociales que l'on peut prendre la mesure du phénomène. En effet, dans les villes où nous avons souligné la présence importante de groupes impliqués dans les activités dramatiques, on remarque que les jeunes gens sont associés à ces représentations, dans le but, nous semble-t-il, d'acquérir au sein de ces groupes festifs les compétences qu'ils seront amenés à utiliser dans leurs futures fonctions d'élites urbaines. Si l'on prend le cas des compagnies joyeuses des villes francophones du sud des Pays-Bas, l'hypothèse a longtemps été évoquée qu'il s'agissait de groupes de jeunesse sur le modèle des abbayes de jeunesse villageoises ${ }^{28}$. Il faut revenir aujourd'hui sur ce calque du modèle villageois aux organisations urbaines, car on constate en effet d'après les documents d'archives mentionnant l'identité des chefs de compagnies que les cadres de ces groupes sont parfois des hommes plus âgés, déjà établis dans un métier et une situation maritale ${ }^{29}$. Ceci n'est pas étonnant quand on sait que cette charge, bien que limitée à un an, entraîne des dépenses assez importantes et doit donc revenir à un homme disposant déjà d'un certain confort financier, sans même évoquer l'importance symbolique de la fonction dans ses rapports privilégiés avec la ville. On peut donc avancer l'hypothèse que ces groupes présentent une certaine mixité sur le plan de l'âge des compagnons et fonctionnent selon un système de transmission des savoirs et des pratiques en leur sein, des membres les plus âgés aux membres les plus jeunes. À travers les pratiques festives impliquant l'organisation de célébrations à l'échelle de la ville et nécessitant des contacts avec les villes voisines, les jeunes gens peuvent ainsi se former à des tâches aussi diverses que la gestion matérielle et financière des spectacles (organisation) et la prise de parole en public (représentation), sans oublier la défense symbolique de l'identité de la ville (déplacements). C'est ce qui pourrait expliquer que l'on retrouve dix à vingt ans plus tard des chefs de compagnies joyeuses à des postes-clés tels que l'échevinage de leur ville (c'est le cas à Cambrai, notamment ${ }^{30}$ ). Le même type de raisonnement peut être tenu pour des groupes aussi variés que la Basoche, les Puys ou les rederijkers néerlandophones ${ }^{31}$.

28. Voir par exemple GrinBerg, 1974. Nathalie Zemon Davis a cependant montré dès la même époque que la filiation entre campagnes et villes était moins évidente qu'il n'y paraissait : voir DAVIS, 1971. Voir aussi Rossiaud, 1976.

29. Il est d'ailleurs significatif que, parmi les noms de ces compagnies joyeuses, seule une minorité renvoie à la jeunesse : la plupart évoquent soit des notions que nous jugeons ironiques et uniquement invoquées dans un but festif (pauvreté et folie), soit des notions renvoyant à la concorde sociale (joie, amitié, « plaisance », etc.).

30. DurieuX, 1883 ; LAVÉANT, 2011c, p. 59-63 et 70-74.

31. Nous renvoyons aux articles traitant de cette question pour différents pays ou régions linguistiques dans Van Dixhoorn et Speakman Sutch, 2008. 
Il faut à cette occasion souligner que l'enquête ne doit pas se limiter aux manifestations théâtrales proprement dites, mais que, pour comprendre ce mécanisme de formation sociale, on doit également prendre en considération d'autres formes de prise de parole en public proches, telles que la récitation de poèmes (pratiquée au même titre que la représentation de pièces de théâtre par les rederijkers, par exemple). On voit ainsi dans les Puys du Nord-Ouest de la France que les jeunes gens peuvent être associés à certaines parties des concours poétiques annuels. Ainsi, au Puy de Rouen, le concours comprend une catégorie spécifique de l'épigramme latine, dont certains indices laissent à penser qu'elle est d'abord pratiquée par ceux en contact quotidien avec cette langue, membres du clergé et maîtres d'école, voire par leurs élèves. Contrairement aux autres séances du Puy, la session de lecture des épigrammes est placée le lundi matin, ce qui pourrait correspondre à un désir d'y faire assister les écoliers, soit qu'ils y participent de façon active, soit qu'ils écoutent les œuvres de leurs condisciples ou de leurs maîtres. Dans les deux cas, la composition d'épigrammes latines répondrait donc à un but didactique comparable à l'exercice dramatique : enseigner aux élèves l'art de la déclamation et de la prise de parole en public, en plus de la composition latine ${ }^{32}$. On peut comparer cette situation à ce qu'on constate dans les Pays-Bas néerlandophones, dans lesquels les chambres de rhétorique jouent un rôle similaire d'enseignement informel pour les plus jeunes membres ${ }^{33}$. En ce sens, donc, on peut véritablement parler d'institutions et de groupes sociaux qui prennent le relais de l'école pour poursuivre, par d'autres moyens, la formation au sens large des jeunes gens.

Il existe un réel intérêt à renouveler l'étude de l'éducation et de la formation aux métiers de la parole à la fin du Moyen Âge en passant par le biais d'une réflexion sur la rencontre de deux objets méritant une attention soutenue : les petites et grandes écoles, restées dans l'ombre des études sur l'université et la formation professionnelle, et le théâtre, considéré jusqu'à une période relativement récente comme un parent pauvre de la littérature médiévale. Or on voit bien que c'est grâce à une réévaluation de l'importance des activités dramatiques en milieu scolaire que l'on peut éclairer des aspects encore trop peu étudiés et compris de la formation des élèves, puis des jeunes gens en milieu urbain. Certes, le théâtre comme moyen pédagogique et didactique reste un objet complexe à cerner, du fait des lacunes des sources d'archives et des textes littéraires. Cependant, il est évident qu'il constitue un terrain de recherche essentiel pour comprendre les dynamiques qui traversent les mondes de l'enseignement, et à plus large échelle, l'ensemble de la société de la fin du Moyen Âge et du début de l'époque moderne ${ }^{34}$.

32. Sur les pratiques poétiques du Puy de Rouen, voir HüE, 2002.

33. VAN DiXHOORn, 2008, p. 136-143 et 148-153.

34. Ces perspectives ont également été soulignées par les articles parus dans un dossier récent de la revue Médiévales : voir IogNA-Prat et SMith, dir., 2010. 


\section{LISTE DES RÉFÉRENCES}

Borgnet (Jules), 1868-1869, « Documents relatifs à l'histoire de l'instruction publique à Dinant ( $\mathrm{XVI}^{\mathrm{e}}$ siècle) », Annales de la société archéologique de Namur, t. 10, p. 223-224.

BouhaïK-Gironès (Marie), 2007, Les Clercs de la Basoche et le théâtre comique (Paris, 1420-1550), Paris, Champion.

BouhaÏK-Gironès (M.), 2010, « Le Recueil général des sotties d'Émile Picot ou la construction d'un genre dramatique », dans BouhaïK-Gironès (M.), Dominguez (Véronique) et Koopmans (Jelle), Les Pères du théâtre médiéval. Examen critique de la constitution d'un savoir académique, Rennes, Presses universitaires de Rennes, p. 121-138.

BouHAÏK-Gironès (M.), 2011a, « Les maîtres du vulgaire. Performance oratoire, savoir-faire rhétorique et maîtrise linguistique dans le théâtre de la fin du Moyen Âge ", dans Leroux (Xavier), Vers une poétique du discours dramatique au Moyen Âge, Paris, Champion (Babeliana, t. 13), p. 225-241.

BouHAÏK-Gironès (M.), 2011b, « Sources et problèmes de l'histoire du théâtre dans les collèges à la fin du Moyen Âge », dans Verger (Jacques) et Sohn (Andreas), Les Collèges universitaires en Europe au Moyen Âge et au XVI siècle, Bochum, Winkler (Ouvertures. Perspectives interculturelles en histoire, politique et religion), p. 107-117.

Carolus-Barré (Louis), 1975, « Les écoles capitulaires et les collèges de Soissons au Moyen Âge et au XVI ${ }^{\mathrm{e}}$ siècle ", dans Enseignement et vie intellectuelle (IXe-XVI siècles). Actes $d u$ $95^{\circ}$ congrès national des sociétés savantes, Paris, Bibliothèque nationale, t. 1, p. 123-226.

Dahнао (Yann), 2005 " Enfant-évêque et fête des Fous : un loisir ritualisé pour jeunes clercs ? ", dans Gilomen (Hans-Jörg), Schumacher (Beatrice) et Tissot (Laurent), Temps libre et Loisirs $d u X I V^{e}$ au $X X^{e}$ siècle/Freizeit und Vergnügen : vom 14. bis zum 20. Jahrhundert, Zurich, Chronos (Société Suisse d'histoire économique et sociale, t. 20), p. 33-46.

DainVILle (François de), 1978, L'Éducation des Jésuites (XVI'-XVIII siècles), Paris, Minuit.

DAvis (Nathalie Zemon), 1971, "The Reasons of Misrule : Youth Groups and Charivaris in Sixteenth-Century France », Past and Present, t. 50, p. 41-70.

De Ridder-Symoens (Hilde), 1995a, " Education and Literacy in the Burgundian-Habsburg Netherlands », Canadian Journal of Netherlandic Studies, t. 16, p. 6-21.

De Ridder-Symoens (H.), 1995b, « La sécularisation de l'enseignement aux anciens Pays-Bas au Moyen Âge et à la Renaissance », dans Duvosquel (Jean-Marie) et Thoen (Erik), Peasants and Townsmen in Medieval Europe. Studia in honorem Adriaan Verhulst, Gand, SnoeckDucaju \& Zoon, p. 721-737.

De Ridder-Symoens (H.), 2004, « The Changing Face of Centres of Learning, 1400-1700 », dans MacDonald (Alasdair) et Twomey (Michael J.), Schooling and Society. The Ordering and Reordering of Knowledge in the Western Middle Ages, Leuven, Peeters, p. 115-138.

Derville (Alain), 1984, «L'alphabétisation du peuple à la fin du Moyen Âge », Revue du Nord, t. 76, n 261-262, p. 761-776.

Desportes (Pierre), 1975, "L'enseignement à Reims aux XIII et XIV ${ }^{\mathrm{e}}$ siècles », dans Enseignement et vie intellectuelle (IX $X^{e}-X I V^{e}$ siècles). Actes du $95^{e}$ congrès national des sociétés savantes, Paris, Bibliothèque nationale, t. 1, p. 107-122.

Doudet (Estelle), 2011, « Pédagogie de l'énigme, le Lyon Marchant de Barthélemy Aneau (1541)», Cahiers de recherches médiévales et humanistes, t. 22, p. 395-411.

Durieux (Achille), 1883, «Le théâtre à Cambrai avant et après 1789 », Mémoires de la société d'émulation de Cambrai, t. 39, p. 5-241.

Ferrand (Mathieu), 2009 « Le théâtre des collèges, la formation des étudiants et la transmission des savoirs aux Xv et XIV siècles », Camenulae, t. 3 (http://www.paris-sorbonne.fr/IMG/pdf/ Mathieu_2.pdf). 
Ferrand (M.), 2010, "Le théâtre des collèges au début du XVI ${ }^{\mathrm{e}}$ siècle : les Dialogi (1530) de Johannes Ravisius Textor », Bibliothèque d'Humanisme et Renaissance, t. $72, \mathrm{n}^{\circ} 2$, p. 337-368.

Ferrand (M.), 2011, « Le théâtre de Barthélemy Aneau : écriture dramatique et pédagogie de l'actio », Cahiers de recherches médiévales et humanistes, t. 22, p. 363-378.

GrinBERG (Martine), 1974, «Carnaval et société urbaine XIV - XVI ${ }^{\mathrm{e}}$ siècles : le royaume dans la ville », Ethnologie française, t. 4, p. 215-244.

Guilleray (Pierre-Emmanuel), 2002, La Fête des Fous dans le Nord de la France (XIV-XVI siècles), Thèse de l'école des Chartes, Paris.

HirSCHAUER (Charles), 1907-1911, « Textes intéressant l'histoire de l'enseignement à SaintOmer, Aire, Hesdin (1569-1570) », Bulletin de la société des antiquaires de la Morinie, t. 12, p. 494-520.

HüE (Denis), 2002, La Poésie palinodique à Rouen (1486-1550), Paris, Champion.

Hummelen (Willem), 1989, « Toneel op de kermis, van Bruegel tot Bredero », Oud Holland, t. 103 , p. 1-45.

Iogna-Prat (Dominique) et Sмith (Darwin), dir., 2010, Théâtres du Moyen Âge. Textes, images et performances, dossier thématique de Médiévales, t. 59.

Knight (Alan E.), 1996 « Beyond Misrule : Theatre and the Socialization of Youth in Lille », Research Opportunities in Renaissance Drama, t. 35, p. 73-84.

Koopmans (Jelle) et Verhuyck (Paul), 1987, «François Villon et le Sermon de Saint Belin », dans ID., Sermon joyeux et truanderie, Villon-Nemo-Ulespiègle, Amsterdam, Rodopi, p. 9-85.

La Fons de Mélicoce (Albert de), 1848, « Extraits de chartes et autres documents originaux concernant les jeux de personnages, mystères, etc., exécutés dans la ville de Béthune et autres lieux de la Flandre pendant le $\mathrm{Xv}^{\mathrm{e}}$ et le $\mathrm{xvI}^{\mathrm{e}}$ siècle », Documents historiques inédits tirés des collections manuscrites de la Bibliothèque royale et des archives ou des bibliothèques des départements, t. 4, éd. Jacques-Joseph Champollion-Figeac, Paris, Imprimerie de l'Institut de France, p. 320-348.

La Fons de Mélicocq (A. de), 1886-1887, « Les artistes dramatiques des provinces de la Flandre et de l'Artois aux $\mathrm{XIV}^{\mathrm{e}}, \mathrm{XV}^{\mathrm{e}}$ et $\mathrm{XVI}^{\mathrm{e}}$ siècles », Mémoires de la société des antiquaires de la Morinie, t. 20, p. 343-454.

LAVÉANT (Katell), 2011a, "Contexte et réception du théâtre scolaire de Noël : de François Briand à Barthélemy Aneau », Cahiers de recherches médiévales et humanistes, t. 22, p. 379-393.

LAVÉANT (K.), 2011b, « L'apprentissage par le théâtre : quatre pièces scolaires pour l'Avent de François Briand (Le Mans, 1512) », dans Leroux (Xavier), Vers une poétique du discours dramatique au Moyen Âge, Paris, Champion (Babeliana, t.13), p. 243-264.

LAVÉANT (K.), 2011c, Un théâtre des frontières (XV'e-XVI siècles), Orléans, Paradigme.

Olaizola Sanchez (Ruth), 2005, Les Jésuites au théâtre dans l'Espagne du Siècle d'Or : théories et pratiques, 1588-1689, Thèse de doctorat, Paris, École des hautes études en sciences sociales.

PiBIRI (Eva), 1998, Sous la férule du maître. Les écoles d'Yverdon (XIV'-XVI siècles), Lausanne, Cahiers Lausannois d'histoire médiévale, t. 23.

Picoт (Émile), s.d., Répertoire des villes, Paris, Bibliothèque nationale de France (ms. fr. n.a.f. 12633-12634).

Rossiaud (Jacques), 1976, « Fraternités de jeunesse et niveaux de culture dans les villes du Sud-Est à la fin du Moyen Âge », Cahiers d'histoire, t. 1-2, p. 67-102.

Rousse (Michel), 2004, «L'espace scénique des farces », La Scène et les tréteaux, le théâtre de la farce au Moyen Âge, Orléans, Paradigme, p. 93-102. 
VALEntin (Jean-Marie), 2001, Les Jésuites et le théâtre (1554-1680). Contribution à l'histoire culturelle $d u$ monde catholique dans le Saint-Empire romain germanique, Paris, Desjonquères.

VAN DiXHOORN (Arjan), 2008, « Chambers of Rhetoric : Performative Culture and Literary Sociability in the Early Modern Northern Netherlands », dans Van Dixhoorn et Speakman Sutch, p. 119-157.

Van Dixhoorn (Arjan) et Speakman Sutch (Susie), 2008, The Reach of the Republic of Letters. Literary and Learned Societies in Late Medieval and Early Modern Europe, Leiden, Brill.

Verger (Jacques), 1995, Les Universités françaises au Moyen Âge, Leiden, Brill.

Verger (J.), 1999, Culture, enseignement et société en Occident aux XII et XIII siècles, Rennes, Presses universitaires de Rennes. 